\title{
CENTERS FOR THE KUKLES HOMOGENEOUS SYSTEMS WITH ODD DEGREE
}

\author{
JAUME GINÉ ${ }^{1}$, JAUME LLIBRE ${ }^{2}$ AND CLAUDIA VALLS ${ }^{3}$
}

\begin{abstract}
For the polynomial differential system $\dot{x}=-y, \dot{y}=$ $x+Q_{n}(x, y)$, where $Q_{n}(x, y)$ is a homogeneous polynomial of degree $n$ there are the following two conjectures done in 1999. (1) Is it true that the previous system for $n \geq 2$ has a center at the origin if and only if its vector field is symmetric about one of the coordinate axes? (2) Is it true that the origin is an isochronous center of the previous system with the exception of the linear center only if the system has even degree? We prove both conjectures for all $n$ odd.
\end{abstract}

\section{Introduction And StATEMEnt of the main RESUlts}

Kukles [11] in 1944 examined the conditions under which the origin for the differential system of the form

(1) $\dot{x}=-y, \quad \dot{y}=x+a_{1} x^{2}+a_{2} x y+a_{3} y^{2}+a_{4} x^{3}+a_{5} x^{2} y+a_{6} x y^{2}+a_{7} y^{3}$,

is a center. For long time it had been thought that the conditions given by Kukles were necessary and sufficient conditions, but some new cases have been found, see $[2,4,10]$. In [4] the center problem for the class of system (1) with $a_{7}=0$ (reduced Kukles system) was resolved, moreover it was shown that at most five limit cycles bifurcate from the origin. In [12] it was solved the center problem for system (1) in the case $a_{2}=0$ and it was shown that at most six limit cycles bifurcate from the origin, see also [13]. The first complete solution of the center-focus problem for Kukles' system (1) was obtained in [13]. In [16] it was also given the complete solution using the Cherkas' method of passing to a Liénard equation, see also the works $[14,17]$. The study of this family exhibits properties and issues which are important in the problem of the full classification of cubic systems with a center.

In this paper we continue the characterization of the centers for a linear center perturbed by homogeneous polynomials, i.e., systems of

2010 Mathematics Subject Classification. Primary 34C05. Secondary 37C10.

Key words and phrases. Integrability, complex center-focus problem, Lyapunov constants, Bautin method. 
the form

$$
\dot{x}=-y, \quad \dot{y}=x+Q_{n}(x, y),
$$

where $Q_{n}(x, y)$ is a homogeneous polynomial of degree $n$, i.e.

$$
Q_{n}(x, y)=\sum_{j=0}^{n} c_{j} x^{j} y^{n-j}, \quad c_{j} \in \mathbb{R} .
$$

These systems are called Kukles homogeneous systems, see [8]. Another important problem is to characterize the isochronous centers of systems of the form (2). We recall that a center is isochronous if all periodic solutions have the same constant period. For a survey about the isochronicity see for instance [18] and the bibliography therein. In [18] and in relation to systems of the form (2) the following two open problems are established.

Conjecture 1. Is it true that a system (2) with nonlinearities of degree higher than two has a center at the origin if and only if its vector field is symmetric about one of the coordinate axes?

Conjecture 2. Is it true that the origin is an isochronous center of system (2) with the exception of the linear center only if the system has even degree and is reduced to system

$$
\dot{x}=-y, \quad \dot{y}=x+x^{2 m-1} y\left(x^{2}+y^{2}\right),
$$

after a change of variables an a possible scaling of the time?

From previous works, see for instance [18], it is known that system (2) for $n=2$ and $n=3$ has no isochronous centers at the origin. Moreover in [18] it is proved that there is exactly one isochronous system for $n=4$ which corresponds to system (4) with $m=1$, and if $n=5$ or $n=7$ then the origin never can be an isochronous center. In [8] it is given a positive answer to the Conjecture 1 for $n=4$ and $n=5$. In this paper we go further in the study of these two problems. In this paper we prove both conjectures for $n \geq 5$ odd.

We remark that there are very few results characterizing centers for polynomial systems of arbitrary degree. One of these results is due to Christopher [3] who characterizes the centers for the Liénard polynomial differential systems of arbitrary degree. In the next result we characterize all the center of the polynomial differential system (2) for degree $n \geq 7$ odd. 
Theorem 1. System (2) with $n \geq 7$ odd has a center at the origin if and only if $c_{2 j}=0$ for $j=0, \ldots,(n-1) / 2$.

Note that Theorem 1 is equivalent to say that system (2) with $n \geq 7$ odd has a center at the origin if and only if it is invariant under the change of variables $(x, y, t) \rightarrow(-x, y,-t)$ and consequently the phase portrait of the system is symmetric to the $y$-axis. So Theorem 1 proves the Conjecture 1 for $n$ odd.

Instead of proving Theorem 1 we will prove a more general result that will lead to the proof of Theorem 1.

Consider the following system

$$
\dot{x}=-y, \quad \dot{y}=x+\left(x^{2}+y^{2}\right)^{(d-n) / 2} Q_{n}(x, y),
$$

where $Q_{n}(x, y)$ is the homogeneous polynomial of degree $n$, given in (3) and $d \geq n$ is odd.

Theorem 2. System (5) with $n \geq 5$ odd and $d \geq n$ odd has a center at the origin if and only if $c_{2 j}=0$ for $j=0, \ldots,(n-1) / 2$.

Note that Theorem 2 clearly implies Theorem 1 . The proof of Theorem 2 will be done by induction over $n$ and is given in section 2 .

The second main result in the paper is the following, which proves Conjecture 2 for $n$ odd.

Theorem 3. System (2) with $n \geq 7$ odd has no isochronous centers.

Instead of proving Theorem 3 we will prove a more general result that will lead to the proof of Theorem 3.

Consider again system (5). In view of Theorem 2 in order that system (5) has a center at the origin we must have $c_{2 j}=0$ for $j=$ $0, \ldots,(n-1) / 2$. Hence, system (5) with a center at the origin can be written as

$$
\dot{x}=-y, \quad \dot{y}=x+\left(x^{2}+y^{2}\right)^{(d-n) / 2} x \sum_{j=0}^{(n-1) / 2} c_{2 j+1} x^{2 j} y^{n-2 j} .
$$

We will prove the following theorem

Theorem 4. System (6) with $n \geq 5$ odd and $d \geq n$ odd has no isochronous centers.

Note that Theorem 4 clearly implies Theorem 3. The proof of Theorem 4 will be done by induction over $n$ and is given in section 3 . 


\section{Proof of Theorem 2}

In this section we prove Theorem 2 by induction over $n$. We start with the case $n=5$. To prove Theorem 2 when $n=5$, we have to prove the following result.

Proposition 5. System (5) with $n=5$ and $d \geq 5$ odd has a center at the origin if and only if $c_{2 j}=0$ for $j=0,1,2$.

Proof. We first prove sufficiency. If $c_{2 j}=0$ for $j=0,1,2$ then system (5) becomes

$$
\dot{x}=-y, \quad \dot{y}=x+\left(x^{2}+y^{2}\right)^{(d-5) / 2} x\left(c_{1} y^{4}+c_{3} x^{2} y^{2}+c_{5} x^{4}\right) .
$$

System (7) is invariant under the symmetry $(x, y, t) \mapsto(-x, y,-t)$. Hence it is invariant with respect to the $y$-axis and it is clear that in this situation system (7) has a center at the origin.

Now we shall prove necessity. To do that we first write the system is complex notation of the form

(8)

$$
\dot{z}=i z+(z \bar{z})^{\frac{d-5}{2}}\left(A_{1} z^{5}+A_{2} z^{4} \bar{z}+A_{3} z^{3} \bar{z}^{2}+A_{4} z^{2} \bar{z}^{3}+A_{5} z \bar{z}^{4}+A_{6} \bar{z}^{5}\right),
$$

where $z=x+i y, d \geq 5$ is an arbitrary odd integer, and

$$
\begin{gathered}
A_{1}=a_{1}+i a_{2}, A_{2}=a_{3}+i a_{4}, A_{3}=a_{5}+i a_{6}, \\
A_{4}=a_{7}+i a_{8}, A_{5}=a_{9}+i a_{10}, A_{6}=a_{11}+i a_{12},
\end{gathered}
$$

where $a_{i} \in \mathbb{R}$. Now we write system ( 8 in the real variables $(x, y)$ and impose that it has the form (5), this implies that

$$
\begin{aligned}
a_{7} & =-a_{5}, \quad a_{8}=a_{6}, \quad a_{9}=-a_{3}, \\
a_{10} & =a_{4}, \quad a_{11}=-a_{1}, \quad a_{12}=a_{2} .
\end{aligned}
$$

Now we write system (5) with $n=5$ and satisfying conditions (9) in polar coordinates, i.e., doing the change of variables $x=r \cos \theta$ and $y=r \sin \theta$, and we obtain

$$
\dot{r}=F(\theta) r^{d}, \quad \dot{\theta}=1+G(\theta) r^{d-1},
$$

where $F(\theta)$ and $G(\theta)$ are the homogeneous trigonometric polynomials

$$
\begin{aligned}
F(\theta)= & 2 \sin \theta\left(a_{6} \cos \theta+a_{4} \cos 3 \theta+a_{2} \cos 5 \theta+a_{5} \sin \theta\right. \\
& \left.+a_{3} \sin 3 \theta+a_{1} \sin 5 \theta\right) \\
G(\theta)= & a_{6}+\left(a_{4}+a_{6}\right) \cos 2 \theta+\left(a_{2}+a_{4}\right) \cos 4 \theta+a_{2} \cos 6 \theta \\
& +\left(a_{3}+a_{5}\right) \sin 2 \theta+\left(a_{1}+a_{3}\right) \sin 4 \theta+a_{1} \sin 6 \theta
\end{aligned}
$$


In order to determine the necessary conditions to have a center we propose the Poincaré series

$$
H(r, \theta)=\sum_{n=2}^{\infty} H_{n}(\theta) r^{n}
$$

where $H_{2}(\theta)=1 / 2$ and $H_{n}(\theta)$ are homogeneous trigonometric polynomials respect to $\theta$ of degree $n$. Imposing that this power series is a formal first integral of system (10) we obtain

$$
\dot{H}(r, \theta)=\sum_{k=2}^{\infty} V_{2 k} r^{2 k}
$$

where $V_{2 k}$ are in fact the Poincaré-Liapunov constants that depend on the parameters of system (8). Due to the Hilbert Basis theorem, the ideal $J=<V_{2}, V_{4}, \ldots>$ generated by the Poincaré-Liapunov constants is finitely generated, i.e. there exist $W_{1}, W_{2}, \ldots, W_{k}$ in $J$ such that $J=<W_{1}, W_{2}, \ldots, W_{k}>$. This set of generators is called a basis of $J$ and the conditions $W_{j}=0$ for $j=1, \ldots, k$ provide a finite set of necessary conditions to have a center. The set of coefficients for which all the Poincaré-Liapunov constants $V_{2 k}$ vanish is called the complex center variety of the family and it is an algebraic set. First we determine a number of Poincaré-Liapunov constants assuming that inside this number there is the set of generators. The next step is to decompose this algebraic set into its irreducible components. We must to use a computer algebra system. The computational tool which we use is the routine minAssGTZ [5] of the computer algebra system SINGULAR [9] which is based on the Gianni-Trager-Zacharias algorithm [7]. The computations in this case can be completed in the field of rational numbers. Hence, all the points of the center variety have been found. That is, we know that all the encountered points belong to the decomposition of the center variety and we do know that the given decomposition is complete.

We have compute $V_{2 k}$ for $k=1, \ldots, 7$. The decomposition of the ideal $J_{7}=<V_{2}, V_{4}, \ldots, V_{14}>$ gives a unique case which is $a_{1}=a_{3}=$ $a_{5}=0$ and we obtain system (7). This completes the proof of the proposition.

It follows from Proposition 5 that Theorem 2 is true for $n=5$ and $d \geq 5$ odd. Now assume it is true for $n=5,7, \ldots, \ell$ with $\ell \geq 5$ odd and we shall prove it for $n=\ell+2$. We thus have that system

$$
\dot{x}=-y, \quad \dot{y}=x+\left(x^{2}+y^{2}\right)^{(d-\ell) / 2} \sum_{j=0}^{\ell} c_{j} x^{j} y^{\ell-j}, \quad c_{j} \in \mathbb{R}
$$


with $d \geq \ell$ odd has a center at the origin if and only if $c_{2 j}=0$ for $j=0, \ldots,[(\ell-1) / 2]$. Now we consider the system

$$
\dot{x}=-y, \quad \dot{y}=x+\left(x^{2}+y^{2}\right)^{(d-\ell-2) / 2} \sum_{j=0}^{\ell+2} c_{j} x^{j} y^{\ell+2-j}, \quad c_{j} \in \mathbb{R} .
$$

First we prove sufficiency. If $c_{2 j}=0$ for $j=0, \ldots[(\ell+1) / 2]$ then system (13) becomes

$$
\dot{x}=-y, \quad \dot{y}=x+\left(x^{2}+y^{2}\right)^{(d-\ell-2) / 2} x \sum_{j=0}^{(\ell+1) / 2} c_{2 j+1} x^{2 j} y^{\ell+1-2 j} .
$$

Since system (14) is invariant under the symmetry $(x, y, t) \mapsto(-x, y,-t)$, it is clear that in this situation system (14) has a center at the origin.

Now we shall prove necessity. Note that we can write

$$
\sum_{j=0}^{\ell+2} c_{j} x^{j} y^{\ell+2-j}=\sum_{j=0}^{(\ell+1) / 2} c_{2 j} x^{2 j} y^{\ell+2-2 j}+\sum_{j=0}^{(\ell+1) / 2} c_{2 j+1} x^{2 j+1} y^{\ell+1-2 j}
$$

Dividing the first summand on the right-hand side of (15) by $x^{2}+y^{2}$ we get

$$
\sum_{j=0}^{(\ell+1) / 2} c_{2 j} x^{2 j} y^{\ell+2-2 j}=\left(x^{2}+y^{2}\right) \sum_{j=0}^{(\ell-1) / 2} d_{2 j} x^{2 j} y^{\ell-2 j}+d_{\ell+1} y^{\ell+2},
$$

and dividing the second summand on the right-hand side of (15) by $x^{2}+y^{2}$ we get

$$
\sum_{j=0}^{(\ell+1) / 2} c_{2 j+1} x^{2 j+1} y^{\ell+1-2 j}=\left(x^{2}+y^{2}\right) \sum_{j=0}^{(\ell-1) / 2} d_{2 j+1} x^{2 j+1} y^{\ell-1-2 j}+d_{\ell+2} x y^{\ell+1} .
$$

Hence it follows from (15) that

$$
\begin{aligned}
\sum_{j=0}^{\ell+2} c_{j} x^{j} y^{\ell+2-j}= & \left(x^{2}+y^{2}\right) \sum_{j=0}^{(\ell-1) / 2} d_{2 j} x^{2 j} y^{\ell-2 j}+d_{\ell+1} y^{\ell+2} \\
& +\left(x^{2}+y^{2}\right) \sum_{j=0}^{(\ell-1) / 2} d_{2 j+1} x^{2 j+1} y^{\ell-1-2 j}+d_{\ell} x y^{\ell+1} \\
= & \left(x^{2}+y^{2}\right) \sum_{j=0}^{\ell} d_{j} x^{j} y^{\ell-j}+d_{\ell+1} y^{\ell+2}+d_{\ell+2} x y^{\ell+1} .
\end{aligned}
$$


Thus we write (13) as

$$
\dot{x}=-y,
$$

(18) $\dot{y}=x+\left(x^{2}+y^{2}\right)^{(d-\ell) / 2} \sum_{j=0}^{\ell} d_{j} x^{j} y^{\ell-j}+d_{\ell+1}\left(x^{2}+y^{2}\right)^{(d-\ell-2) / 2} y^{\ell+2}$

$$
+d_{\ell+2}\left(x^{2}+y^{2}\right)^{(d-\ell-2) / 2} x y^{\ell+1}
$$

with $d_{j} \in \mathbb{R}$ for $j=0, \ldots, \ell+2$.

Now assume that system (18) has a center at the origin and some $d_{2 j} \neq 0$ for $j=0, \ldots,(\ell-1) / 2$. Since system (18) has a center at the origin, there exists a first integral $H=H_{d_{\ell+1}, d_{\ell+2}}(x, y)$ defined in a neighborhood of the origin which is analytic in the parameters $d_{\ell+1}$ and $d_{\ell+2}$. Hence, if we expand $H_{d_{\ell+1}, d_{\ell+2}}(x, y)$ in the parameters $d_{\ell+1}$ and $d_{\ell+2}$ we see that system (18) restricted to $d_{\ell+1}=d_{\ell+2}=0$ has the analytic first integral $H_{0,0}(x, y)$ defined in a neighborhood of the origin. This in particular implies that system (18) restricted to $d_{\ell+1}=d_{\ell+2}=0$, i.e., system

$$
\dot{x}=-y, \quad \dot{y}=x+\left(x^{2}+y^{2}\right)^{(d-\ell) / 2} \sum_{j=0}^{\ell} d_{j} x^{j} y^{\ell-j}
$$

has a center at the origin. But by hypothesis (see (12)) this is not possible since system (19) has a center at the origin if and only if $d_{2 j}=0$ for $j=0, \ldots,(\ell-1) / 2$.

In short, if system (18) has a center at the origin then $d_{2 j}=0$ for $j=0, \ldots,(\ell-1) / 2$. We then have that system (18) becomes

$$
\dot{x}=-y,
$$

$$
\begin{aligned}
\dot{y}= & x+\left(x^{2}+y^{2}\right)^{(d-\ell) / 2} \sum_{j=0}^{(\ell-1) / 2} d_{2 j+1} x^{2 j+1} y^{\ell-1-2 j} \\
& +d_{\ell+1}\left(x^{2}+y^{2}\right)^{(d-\ell-2) / 2} y^{\ell+2}+d_{\ell+2}\left(x^{2}+y^{2}\right)^{(d-\ell-2) / 2} x y^{\ell+1},
\end{aligned}
$$

that we write as

$$
\dot{x}=-y, \quad \dot{y}=x+P_{\ell+2}(x, y)+d_{\ell+1}\left(x^{2}+y^{2}\right)^{(d-\ell-2) / 2} y^{\ell+2},
$$

where

$$
\begin{aligned}
P_{\ell+2}(x, y)= & \left(x^{2}+y^{2}\right)^{(d-\ell) / 2} \sum_{j=0}^{(\ell-1) / 2} d_{2 j+1} x^{2 j+1} y^{\ell-1-2 j} \\
& +d_{\ell+2}\left(x^{2}+y^{2}\right)^{(d-\ell-2) / 2} x y^{\ell+1} .
\end{aligned}
$$


Consider now the system

$$
\dot{x}=-y, \quad \dot{y}=x+P_{\ell+2}(x, y) .
$$

Note that system (22) is invariant under the symmetry $(x, y, t) \mapsto$ $(-x, y,-t)$, and this symmetry insures that origin is a center. Hence, system (22) has a center at the origin. Moreover, the cross product between the vector fields to systems (22) and (21) is locally positive or locally negative in a neighborhood of the origin unless $d_{\ell+1}$ vanishes. Since (22) has a center, (21) can only have a center if $d_{\ell+1}=0$. Hence, a necessary condition for system (13) to have a center at the origin is that $c_{2 j}=0$ for $j=0, \ldots,(\ell+1) / 2$ (see also equations (15) and (16)). This concludes the proof of the induction process and completes the proof of Theorem 2 .

\section{Proof of Theorem 4}

In this section we prove Theorem 4 by induction over $n$. We start with the case $n=5$. To prove Theorem 4 when $n=5$, we have to prove the following result.

Proposition 6. System (6) with $n=5$ and $d \geq 5$ odd, has no isochronous centers.

Proof. System (10) is equivalent to

$$
\frac{d r}{d \theta}=\frac{F(\theta) r^{d}}{1+G(\theta) r^{d-1}} .
$$

It is clear that equation (23) is well defined in a sufficient small neighborhood of the origin. The transformation $(r, \theta) \rightarrow(\rho, \theta)$ introduced by Cherkas [1] defined by

$$
\rho=\frac{r^{d-1}}{1+G(\theta) r^{d-1}}, \quad \text { whose inverse is } \quad r=\frac{\rho^{1 /(d-1)}}{(1-\rho G(\theta))^{1 /(d-1)}},
$$

is a diffeomorphism from the region $\dot{\theta}>0$ into its image. If we transform equation (23) using the transformation (24), we obtain the Abel equation

$$
\frac{d \rho}{d \theta}=-(d-1) G(\theta) F(\theta) \rho^{3}+\left[(d-1)\left(F(\theta)-G^{\prime}(\theta)\right] \rho^{2} .\right.
$$

The solution $\rho\left(\theta, \rho_{0}\right)$ of $(25)$ satisfying that $\rho\left(0, \rho_{0}\right)=\rho_{0}$ can be expanded in a convergent series of $\rho_{0} \geq 0$ sufficiently small of the form

$$
\rho\left(\theta, \rho_{0}\right)=\rho_{1}(\theta) \rho_{0}+\rho_{2}(\theta) \rho_{0}^{2}+\rho_{3}(\theta) \rho_{0}^{3}+\cdots
$$

with $\rho_{1}(\theta)=1$ and $\rho_{k}(0)=0$ for $k \geq 2$. Let $P:\left[0, \tilde{\rho}_{0}\right] \rightarrow \mathbb{R}$ be the Poincaré return map defined by $P\left(\tilde{\rho}_{0}\right)=\rho\left(2 \pi, \tilde{\rho}_{0}\right)$ for a convenient 
$\tilde{\rho}_{0}$. System (8) has a center at the origin if and only if $\rho_{k}(2 \pi)=0$ for every $k \geq 2$. If we assume that $\rho_{2}(2 \pi)=\cdots=\rho_{m-1}(2 \pi)=0$ we say that $v_{m}=\rho_{m}(2 \pi)$ is the $m$-th Poincaré-Liapunov-Abel constant of system (8). Of course the set of coefficients for which all the PoincaréLiapunov-Abel constants $v_{m}$ vanish is the same that the set for which all the Poincaré-Liapunov constants $V_{2 k}$ vanish. If we compute these constants when system (10) has a center then all the $v_{m}$ are null. However we also can to determine which of these centers are isochronous.

From the second equation of (10) we have

$$
T=\int_{0}^{2 \pi} \frac{d \theta}{\dot{\theta}}=\int_{0}^{2 \pi} \frac{1}{1+G(\theta) r(\theta)^{d-1}} d \theta .
$$

Using the change (24) the previous integral becomes

$$
T=\int_{0}^{2 \pi}(1-G(\theta) \rho(\theta)) d \theta=2 \pi-\int_{0}^{2 \pi} G(\theta) \rho(\theta) d \theta,
$$

where $\rho(\theta)=\sum_{j \geq 1} \rho_{j}(\theta) \rho_{0}^{j}$ is the solution given in (26). System (8) has an isochronous center at the origin if the origin is a center and satisfies

$$
\int_{0}^{2 \pi} G(\theta) \rho(\theta) d \theta=\sum_{j \geq 1}\left(\int_{0}^{2 \pi} G(\theta) \rho_{j}(\theta) d \theta\right) \rho_{0}^{j}=0 .
$$

That is $T=\int_{0}^{2 \pi} d \theta / \dot{\theta}=2 \pi-\sum_{j \geq 1} T_{j} \rho_{0}^{j}=2 \pi$, where

$$
T_{j}=\int_{0}^{2 \pi} G(\theta) \rho_{j}(\theta) d \theta
$$

are called the period Abel constants. For system (6) with $n=5$ and $d \geq 5$ odd, the first period Abel constants are

$$
\begin{gathered}
T_{1}=a_{6}, \quad T_{2}=-25 a_{2}^{2}-24 a_{2} a_{4}-27 a_{4}^{2}+a_{2}^{2} d+3 a_{4}^{2} d, \\
T_{3}=625 a_{2}^{3}+1017 a_{2}^{2} a_{4}+900 a_{2} a_{4}^{2}+594 a_{4}^{3}-50 a_{2}^{3} d-84 a_{2}^{2} a_{4} d \\
-69 a_{2} a_{4}^{2} d-135 a_{4}^{3} d+a_{2}^{3} d^{2}+3 a_{2}^{2} a_{4} d^{2}-3 a_{2} a_{4}^{2} d^{2}+9 a_{4}^{3} d^{2} .
\end{gathered}
$$

The decomposition of the ideal generate by the period Abel constants gives a unique case which is $a_{6}=a_{4}=a_{2}=0$, and we obtain that the unique isochronous system is the linear one. This completes the proof of the proposition.

It follows from Proposition 6 that Theorem 4 is true for $n=5$ and $d \geq 5$ odd. Now assume it is true for $n=5,7 \ldots, \ell$ with $\ell \geq 5$ odd and 
we shall prove it for $n=\ell+2$. We thus have that system

$$
\dot{x}=-y, \quad \dot{y}=x+\left(x^{2}+y^{2}\right)^{(d-\ell) / 2} \sum_{j=0}^{(\ell+1) / 2} c_{2 j+1} x^{2 j+1} y^{\ell-1-2 j}
$$

with $d \geq \ell$ odd has no isochronous centers. Now we consider the system

$$
\dot{x}=-y, \quad \dot{y}=x+\left(x^{2}+y^{2}\right)^{(d-\ell-2) / 2} \sum_{j=0}^{(\ell+1) / 2} c_{2 j+1} x^{2 j+1} y^{\ell+1-2 j} .
$$

We will prove that system (30) has no isochronous centers.

As in the proof of Theorem 2 we write

$$
\begin{aligned}
& \sum_{j=0}^{(\ell+1) / 2} c_{2 j+1} x^{2 j+1} y^{\ell+1-2 j}= \\
& \quad=\left(x^{2}+y^{2}\right) \sum_{j=0}^{(\ell-1) / 2} d_{2 j+1} x^{2 j+1} y^{\ell-1-2 j}+d_{\ell+2} x y^{\ell+1}
\end{aligned}
$$

Thus we write (30) as

$$
\begin{aligned}
\dot{x}= & -y, \\
\dot{y}= & x+\left(x^{2}+y^{2}\right)^{(d-\ell) / 2} \sum_{j=0}^{(\ell-1) / 2} d_{2 j+1} x^{2 j+1} y^{\ell-1-2 j} \\
& +d_{\ell+2}\left(x^{2}+y^{2}\right)^{(d-\ell-2) / 2} x y^{\ell+1},
\end{aligned}
$$

with $d_{j} \in \mathbb{R}$ for $j=0, \ldots, \ell+2$.

Now assume that system (32) has an isochronous center and some $d_{2 j+1} \neq 0$ for $j=0, \ldots,(\ell-1) / 2$. Since system (32) has an isochronous center then the period is constant. Since the period is constant, and the solution $r(\theta)$ depends continuously on the parameter $d_{\ell+2}$, we get that $T=T\left(d_{\ell+2}\right)$ is constant and only depends (analytically) on $d_{\ell+2}$. Expanding $T$ in power series in $d_{\ell+2}$ we have that $T(0)$ is constant and is indeed the period of the system

$$
\begin{aligned}
& \dot{x}=-y, \\
& \dot{y}=x+\left(x^{2}+y^{2}\right)^{(d-\ell) / 2} \sum_{j=0}^{(\ell-1) / 2} d_{2 j+1} x^{2 j+1} y^{\ell-1-2 j} .
\end{aligned}
$$

But this implies that system (33) has an isochronous center. But by hypothesis (see (29)) this is not possible since system (33) has an isochronous center if and only if $d_{2 j+1}=0$ for $j=0, \ldots,(\ell-1) / 2$. 
In short, if system (32) has an isochronous center then $d_{2 j+1}=0$ for $j=0, \ldots,(\ell-1) / 2$. We then have that system (32) becomes

$$
\dot{x}=-y, \quad \dot{y}=x+d_{\ell+2}\left(x^{2}+y^{2}\right)^{(d-\ell-2) / 2} x y^{\ell+1} .
$$

We have the following proposition.

Proposition 7. If system (34) has an isochronous center, then $d_{\ell+2}=$ 0 .

Proof. We take polar coordinates $x=r \cos \theta$ and $y=r \sin \theta$ and system (34) takes the form

$$
\dot{r}=k r^{d} \cos \theta \sin ^{2+\ell} \theta, \quad \dot{\theta}=1+d_{\ell+2} r^{d-1} \cos ^{2} \theta \sin ^{1+\ell} \theta .
$$

Next we apply the Cherkas' transformation to arrive to the associated Abel equation (25). Then we compute the solution $\rho\left(\theta, \rho_{0}\right)$ of $(25)$ satisfying that $\rho\left(0, \rho_{0}\right)=\rho_{0}$ up to certain order. Using these expansion and equation (28) we find that the first period Abel constant is

$$
T_{1}=\frac{\left(-1+(-1)^{\ell}\right) d_{\ell+2} \pi^{3 / 2}}{2 \Gamma\left(-\frac{\ell}{2}\right) \Gamma\left(\frac{5+\ell}{2}\right) \sin \left(\frac{\pi \ell}{2}\right)} .
$$

Taking into account that $\ell$ is odd we obtain that $d_{\ell}$ must be zero.

It thus follows from Proposition 7 that a necessary condition in order that system (6) has an isochronous center is that $c_{2 j+1}=0$ for $j=$ $0, \ldots,(\ell+1) / 2$ (see also (31)). But this implies that system (6) becomes

$$
\dot{x}=-y, \quad \dot{y}=x .
$$

This concludes the proof of Theorem 4 .

\section{ACKnowledgements}

The first author is partially supported by a MINECO/FEDER grant number MTM2011-22877 and an AGAUR (Generalitat de Catalunya) grant number 2014SGR 1204. The second author is partially supported by a MINECO/ FEDER grant number MTM2008-03437, an AGAUR grant number 2014SGR 568, ICREA Academia, two FP7-PEOPLE2012-IRSES numbers 316338 and 318999, and FEDER-UNAB10-4E378. The third author is supported by Portuguese National Funds through FCT - Fundação para a Ciência e a Tecnologia within the project PTDC/MAT/117106/2010 and by CAMGSD. 


\section{REFERENCES}

[1] L.A. Cherkas, The number of limit cycles of a certain second order autonumous system (Russian), Differencial'nye Uravnenija 12 (1976), 944-946.

[2] L.A. Cherkas, Conditions for the equation $y y^{\prime}=\sum_{i=0}^{3} p_{i}(x) y^{i}$ to have a centre, Differential Equations 14 (1978), 1133-1138.

[3] C.J. Christopher, An algebraic approach to the classification of centers in polynomial Liénard systems, J. Math. Anal. Appl. 229 (1999), 319-329.

[4] C.J. Christopher, N.G. Lloyd, On the paper of Jin and Wang concerning the conditions for a centre in certain cubic systems, Bull. London Math. Soc. 22 (1990), 5-12.

[5] W. Decker, S.laplagne, G. Pfister, H.A. Schonemann, SinguLAR 3-1 library for computing the prime decomposition and radical of ideals, primdec.lib, 2010.

[6] A. Cima, A. Gasuld, V. Mañosa, F. Mañosas, Algebraic properties of the Liapunov and period constants, Rocky Mountain J. Math. 27 (1997), 471-501.

[7] P. Gianni, B. Trager, G. Zacharias, Gröbner bases and primary decompositions of polynomials, J. Symbolic Comput. 6 (1988) 146-167.

[8] J. Giné, Conditions for the existence of a center for the Kukles homogenenous systems, Comput. Math. Appl. 43 (2002), 1261-1269.

[9] G.M. Greuel, G. Pfister, H.A. Schönemann Singular 3.0 A Computer Algebra System for Polynomial Computations, Centre for Computer Algebra, University of Kaiserlautern (2005). http://www. singular .uni-kl.de.

[10] X. Jin, D. WANG, On the conditions of Kukles for the existence of a centre, Bull. London Math. Soc. 22 (1990), 1-4.

[11] I.S. KukLES, Sur quelques cas de distinction entre un foyer et un centre, Dokl. Akad. Nauk. SSSR 42 (1944), 208-211.

[12] N.G. Lloyd, J.M. PeARson, Conditions for a centre and the bifurcation of limit cycles, in Bifurcations of Planar Vector Fields, J.P. Françoise, R. Roussarie (Eds.), Lecture Notes Math., vol. 1455, Springer, 1990, pp. 230-242.

[13] N.G. Lloyd, J.M. Pearson, Computing centre conditions for certain cubic systems, J. Comp. Appl. Math. 40 (1992), 323-336.

[14] J.M. PeArson, N.G. Lloyd, Kukles revisited: advances in computing techniques, Comput. Math. Appl. 60 (2010), 2797-2805.

[15] V.G. Romanovski, M. PREŠERn, An approach to solving systems of polynomials via modular arithmetics with applications, J. Comput. Appl. Math. 236 (2011), 196-208.

[16] A. P. SAdovskir, Solution of the center and focus problem for a cubic system of nonlinear oscillations, (Russian) Differ. Uravn. 33 (1997), no. 2, 236-244, 286; translation in Differential Equations 33 (1997), 236-244.

[17] A.S. SHubè, On the Kukles and Cherkas center conditions for a cubic system, Differential Equations 29 (1993), 625-627.

[18] E. P. Volokitin, V. V. Ivanov, Isochronicity and Commutation of polynomial vector fields, Siberian Mathematical Journal 40 (1999), 22-37.

1 Departament de Matemàtica, Universitat de Lleida, Avda. Jaume II, 69; 25001 Lleida, Catalonia, Spain

E-mail address: gine@matematica.udl.cat 
2 Departament de Matemàtiques, Universitat Autònoma de Barcelona, 08193 Bellaterra, Barcelona, Catalonia, Spain

E-mail address: jllibre@mat.uab.cat

3 Departamento de Matemática, Instituto Superior Técnico, Av. Rovisco Pais 1049-001, Lisboa, Portugal

E-mail address: cvalls@math.ist.utl.pt 